\title{
Correction to: Testing the moss layer transfer technique on mineral well pads constructed in peatlands
}

\author{
Marie-Eve Gauthier • Line Rochefort $\mathbb{D}$ - Leonie Nadeau - Sandrine Hugron • \\ Bin Xu
}

Published online: 28 May 2018

(C) Springer Science+Business Media B.V., part of Springer Nature 2018

Correction to: Wetlands Ecol Manage

https://doi.org/10.1007/s11273-017-9532-4

In the original publication, the Table 1 was published incorrectly. The correct version of Table 1 is given in this correction. The original article has been corrected.

The original article can be found online at https:// doi.org/10.1007/s11273-017-9532-4.

M.-E. Gauthier · L. Rochefort $(\bowtie) \cdot S$. Hugron Department of Plant Sciences and Centre for Northern Studies, Université Laval, Québec, QC G1V 0A6, Canada e-mail: line.rochefort@fsaa.ulaval.ca

L. Nadeau $\cdot$ B. Xu

NAIT Boreal Research Institute, Peace River,

AB T8S 1R2, Canada 
Table 1 Description of fen plant communities used as source of propagules (donor sites) for the moss layer transfer experiment

\begin{tabular}{|c|c|c|c|c|}
\hline & Treed Rich Fen & Cover & Shrubby Rich Fen & Cover \\
\hline \multicolumn{5}{|l|}{ Plant composition } \\
\hline Trees & Picea mariana & 10 & - & \\
\hline \multirow[t]{6}{*}{ Shrubs } & Vaccinium vitis-idaea & 12 & Salix spp. & 15 \\
\hline & Larix laricina & 9 & Betula glandulosa & 2 \\
\hline & Chamaedaphne calyculata & 8 & & \\
\hline & Empetrum nigrum & 4 & & \\
\hline & Rhododendron groenlandicum & 4 & & \\
\hline & Salix spp. & 4 & & \\
\hline \multirow[t]{3}{*}{ Herbs } & Carex aquatilis & 3 & Carex aquatilis & 7 \\
\hline & Carex tenuiflora & * & Comarum palustre & 2 \\
\hline & & & Carex magellanica ssp. irrigua & 1 \\
\hline \multirow[t]{3}{*}{ Mosses } & Sphagum fuscum & 55 & Sphagnum angustifolium & 30 \\
\hline & Aulacomnium palustre & 3 & Tomentypnum nitens & 15 \\
\hline & & & Aulacomnium palustre & 3 \\
\hline \multicolumn{5}{|l|}{ Water chem. } \\
\hline $\mathrm{pH}$ & 5.7 & & 6.9 & \\
\hline $\mathrm{EC}\left(\mu \mathrm{S} \mathrm{cm}{ }^{-1}\right)$ & 190 & & 98 & \\
\hline $\mathrm{Ca}\left(\mathrm{mg}^{-1}\right)$ & 17 & & 12 & \\
\hline Water table $(\mathrm{cm})$ & $-22.0 \pm 3.2$ & & $-7.0 \pm 3.0$ & \\
\hline
\end{tabular}

Cover is in $\%$ and $*=$ presence. Water table depth was measured five times $(n=5)$ during the summer, the data presented is the average $\pm \mathrm{SD}$ 